\title{
(7)
}

\section{HOW SHALL WE TREAT INCIPIENT CHOLERA.}

By C. Handfield Jones, M.B., F.R.S., Physician to St. Mary's Hospital, etc.

I HAVE somewhat hesitated to reply to Dr. G. Johnson's strictures on the remarks contained in my letter of May 5th, 1866, because I really do not wish to appeur as a partisan, but as a candid inquirer after truth. I do hope that a good many of those who saw much of former epidemics will give us their experience as pithily as Mr. Davis has his in the Medical Times and Gazette, May 5th. To get this information is my chief object. Meanwhile, however, the difficulties which I feel in accepting completely Dr. Johnson's views may be felt by others, and I trust that no harm, and possibly some advantage, may accrue from a temperate exposition of them. Above all things, let us have calm, friendly discussion, and avoid all approach to that unseemly thing,-a doctor's squabble. I notice the several points seriatim.

Admitting the cholera stools to be poisonous, though contra, I may refer to Dr. Goodeve's hospital experience (Reynolds's System of Medicine, vol. i, p. 145), they generally are carried off too rapidly to make it necessary to purge them away; and if otherwise, absorption is for the most part so much in abeyance during the early stages, that the need for removal is much lessened. On the authority of Dr. Johnson's own statements, "that so long as the poison remains in the system, there is going on a rapid manufacture of cholera cathartine, which must and will purge itself away," I do not see how, in the premonitory purging of cholera, there can possibly be any need for castor oil. How active the intestinal clearing out is in such conditions, we may learn from a history given in Annesley's work, in which it is mentioned that a man having swallowed from ten to fifteen minims of peppermint oil on a betel-leaf, passed the leaf per anum, with the next evacuation. No need for any clearing out here. I once had copious rice-water diarrhcea myself after a small dose of Epsom salts, taken as an aperient, and I do think that any further flushing of my canal would have been superfluous, to say the least. Again, I think it most improbable that any amount of evacuation from the blood or bowel frees the system effectually from the poison. Dr. Bence Jones's recent experiments have shown us how intimately absorbed matters penetrate into the tissues, even the non-vascular; and it is surely quite unproved that after any amount of purging the remaining constituents of the body are not just as much tainted (relatively to their size) as they were before, so that reproduction of the poison would always be going on. The intestinal profluvium has not the character of a critical excretion, but of a mere symptomatic. It is much more a mere draining off of liquid from the blood under some peculiar alteration of the capillary walls than a true excretion, and it seems extremely doubtful whether the influence of cell-growth is at all concerned in its production. Admitting, as I am much inclined to do, that the state of collapse is not essentially dependent on the drain of fluid from the blood; yet surely no one can regard the loss as insignificant, or as not likely to be injurious to a weakened system. The analogy of other zymotic diseases,-if I may refer to it,-makes it also unlikely that the intestinal elimination is curative. How long a scarlatina patient continues to be a source of infection after convalescence has commenced is well known, and in other fevers of the same class the same holds true, though probably for a shorter period. I am very much of Dr. Gull's opinion (vide Medical Times and Gazette, April 5, 1862), that recovery from these disorders takes place not by the system being freed from the poison, but from its becoming indifferent to it, - acclimatised, as it were. Dr. Gull's remarks are well worth reading.

To the question, what evidence I have that the cholera poison resembles the malarious? I answer, that my own observation fully coincides with that of various authorities as to the close resemblance of their effects. Annesley says, p. 132, "Here I may at once state, that there seems to be sufficient proof that the situations remarkable for the prevalence of intermittent and remittent fevers are favourable to the action of the efficient cause of the disease upon the system, if not to the development of the cause itself. At Sindwa and Candeish, the disappearance of cholera was succeeded by severe cases of remittent and intermittent fever." Mr. Hutchinson writes, "to me, as to many others, there appears to exist a striking analogy between it (cholera) and fever, both in its intermittent and congestive types. When the cold fit in fever, or collapse in cholera, prove fatal, the similarity is still more striking," p. 66. Dr. Goodeve says, "I have seen people under the influence of malarious poison in Calcutta lie for hours as cold, as pulseless, and as embarrassed in the breathing as in cholera." Trousseau, writing of pernicious fevers, says, "when it is the intestine which suffers, there may ensue a watery diarrhœal flux, analogous in its abundance to that which is poured forth in cholera, and attended with great prostration. In sudoral fever, the sweat, which is cold, comes on sooner than usual, and inundates the surface of the body. The pulse is rapid, small, and weak, the respiration quick and difficult. The fingers look as if macerated; the face becomes livid; and the loss of temperature is so great that it is needful to apply artificial warmth to the patient, who may sink in the first paroxysm" (vol. iii, pp. 431, 429). Surely, such conditions are very like choleraic. Dr. James Bird writes, "so forcibly, indeed, was the resemblance impressed on my mind by many of the cholera cases seen in India, that I could not help regarding the disease as but the cold stage of an intermittent, in which the superadded diarrhcea prevented all febrile reaction.... The immediate causes producing the fatal malady seem connected with epidemic and endemic influences, such as produce intermittent fever, and with those atmospheric changes which at particular periods of the year render it more or less prevalent. Attacks of cholera, when endemic, most commonly happen at the changes of the moon. Remittent and intermittent fevers, with gastro-enteritic affections and diarrhoe, are the most common forms of disease where cholera is prevalent, and partake of the same atonic and congestive character which distinguishes it. These facts justify the inference that malaria is the cause of both diseases, but I will not venture further than to point out their connexion." (London Journal of Medicine, Jan. 1849.) Again, he proceeds, "so forcibly had facts brought this conviction to my mind, that in my cholera reports in 1819 , I pointed out the apparently intimate connexion of the two, stating that several cases had come under my observation where cholera and intermittent fever alternated with each other" (Tract on Cholera). Dr. Charles Bell says that he became acquainted with 
cholera under very favourable circumstances in Edinburgh and London in 1832, 1833, and 1834, and was then stationed in Persia for several years. He had there the opportunity of observing closely the first approaches of the cholera. The disease was ushered in by a regular succession of epidemics, commencing in a fever apparently continued, but by and by assuming more and more the character of a remittent, and this very gradually changed to an intermittent of a quotidian type ; of this, the cold stage gradually became prolonged, and assumed all the appearance of an attack of cholera, and then came the cholera as it has everywhere been known, without any obvious stages or intermission. This again in its turn disappeared, and the epidemic resumed the character of remittent and continued fever for a time. These various changes occupied a period of eighteen months" (Monthly Retrospect of Medical Science, 1849, p. 26). Dr. Cormack, from experience gained in the vicinity of London, near Putney, comes to the following conclusions :-1. Cholera is a fever intimately related to those fevers which depend on malaria. 2. The intermittent or remittent type can be generally recognised in the milder, 'and also not unfrequently (though less distinctly) in the severer cases. 3. The stage of collapse ought to be considered as an aggravated cold stage of the paroxysm of a pernicious fever, which may spontaneously terminate in death or reaction. Dr. Billing holds much the same view. Dr. Prout's observation evidently led him to think that the cause of cholera had an affinity to ordinary malaria, vide p. 23.

I do not wish to make too much of these quotations, but I think it is quite impossible to disregard them. They point to what I may subsequently advert to, viz., the influence of some other element than mere contagion or infection in the production of cholera. My own observation amounts to this: that there existed, during the prevalence of cholera in 1854, in and about Paddington at least, some peculiar general, I will not say atmospheric influence, which manifested itself especially in the way of disordering nervous power. Of this I have no sort of doubt. At Hampstead this did not exist; and the peculiar depression which one felt in the town, was speedily shaken off on the higher elevation. I saw numerous cases of anomalous nerve disorder about and after this period; and $I$ experienced in my own person (Aug. 1854) a remarkable attack, which was certainly much more like ague than cholera. It was preceded by a most forcible presentiment of impending death. The symptoms were severe rigors, chills, great depression, and an intense sensation in the abdomen, as if the bowels were about to act copiously, but no evacuation ensued. I took immediately liquor opii sedativus and chloric ether, and after two doses reaction came on, I lost much of the depression and perspired profusely. The next morning (the attack occurred at night) I felt fairly well, but looked very pale and ashy, and was quite unfit for my usual avocations. During the next six weeks I had several recurrences, but much less severe, and there was an almost constant tendency in the bowels to be over-active. My brainpower was much enfeebled. Going out of town was more beneficial than anything else. As the epidemic ceased, I regained my usual health. Now, I do not say that this disorder was ague any more than it was cholera; but I do think that it resembled malarious poisoning more than anything else. No one of any experience can be unaware how manifold are the forms in which malarious disorder manifests itself, and that the one feature which is common to them all is the remarkable impairment of nervous power. The assanlts of malarious fever are sometimes as sudden and deadly as those of the most intense cho- lera. Intermittency -it is, I think, tolerably certain $\underset{\mathbb{\Phi}}{\mathbb{D}}$ -is much more dependent on the nervous system than on the poison. It has been most marked in: cases of disease of the membranes of the brain with out any malarial intoxication.

It is precisely " from observing their effects upor? the functions and structure of the body" that I amp led to think with others, that the causes of choleras and ague are not essentially different. All intered mittents and remittents probably do not acknowledge precisely the same kind of cause. Dr. Livingstone tells us, that the remittent fevers of the Zambesi are. not cured by any amount of quinine. Let anyon read the account of the fevers of Jerusalem given by Dr. Chaplin (Lancet, Aug., Sept., 1864), and sajw whether he does not think that there must be specifie differences in the exciting causes as well as in the excipients. I believe the experience of most of those. who have witnessed intertropical disease would go to establish the same point. Fevers are often namedo after different places on account of some peculiars effects produced. The malaria at Bussorah and irios the Punjaub has been observed at times to affect als exposed to its influence with vomiting after mealso (Trans. of the Bombay Med. and Phys. Soc., 1853 andP 1854, pp. 213, 216.) The cholera fever briefly dew scribed by Sir R. Martin was evidently a peculiar form of disease, and the cause giving rise to it must have been a modification of the ordinary choleraie influence. The following paragraph from his wellknown work (vide p. 557) has a strong bearing on theo point in question. "Not only was the peculiar fevem here noticed prone to merge into true cholera, but $\exists$ had to remark on three several occasions, in Calcuttao that when the cholera poison was present in a cons centrated form, we were for the time precluded from treating the cases of ordinary remittent fever on Europeans in our usual manner. Moderate blood응 lettings were more liable to be followed by extra ordinary collapse, and purgatives could not be ad $\neq$ ministered over night lest the fever should be changed by morning into a deadly cholera. The state of the system here indicated showed that the fevers, and also the diarrhœas of the country, did, under epidemi or other peculiar influences, merge into the true cho lera, the cholera poison being at the moment the more powerful influence. In times like these, we found it difficult to persuade ourselves that the endemic and the epidemic diseases were absolutely caused by different poisons." Dr. Goodeve says, "it has frequently been noticed that at certain periods of the cholera epidemic, towards its decline, the choleraie diarrhœas pass into a sort of low fever." In speakingo of the Kurrachee epidemic, Mr. Thom says, "as cho lera closed its career, it gradually changed its typer to that of fever; while one out of four or five cases ran into spasmodic cholera, the others would termio nate in fever, and were registered as such, showing. all the characters of a low remittent." It is weln known that the cause of influenza occasionally givesu rise to intermittent fever, which certainly proves that. there must be an affinity between the two. Ye surely the history and symptoms of influenza are very different from those of ague. To me there seems to be quite as much affinity between the causes? of cholera and ague as between those of ague and influenza. The much greater prevalence of malarious affections some years than in others has often been noticed; and it is affirmed by Dr. Metcalfe, in the Essay on Miasmatic Fever, published by the United States Sanitary Commission, p. 212, "that it is not possible to predict the amount of malarious feves that will ensue from any given conditions of heat $\Omega$ moisture, atmospheric or telluric phenomena." The existence of some peculiar general influence, indes 
pendent of contagion during cholera periods, muist, I think, absolutely be admitted, and this influence seems to me to have much affinity to malarious.

Dr. Johnson attempts a reductio ad absurdum of my argument against purging being effectual in removing choleraic poison, because it does not remove malarious in the following,-quinine will not cure cholera ; probably, therefore, it is not a cure for ague. I demur to both positions. Quinine may be, I believe, very useful in some choleraic affections, strengthening and enabling the system to resist the poison, or to prevent a recurrence of a dangerous paroxysm. Annesly recommends the use of bark, with other means tending to conserve vital energy, as a preservative. Quinine will not cure a choleraic paroxysm any more than it will cure an agueish. In both conditions, it is used to fortify the system and enable it to withstand the subsequent assaults, not directly to cure. Its action is analogous in both cases.

With regard to the argument from analogy between cholera and other toxic diseases, I cannot think that Dr. G. Johnson has appreciated it adequately. Analogy is not, I admit, a safe guide, unless we are satisfied of the real likeness of the things which we compare. But he reckons cholera as a zymotic disease; and therefore it may surely be fairly compared with typhoid, typhus, variola, measles, scarlatina, and probably erysipelas. Let us consider whether in these we have any reason to think that a plan of treatment, based on the idea of eliminating the poison from the system by any of the excretory channels (saving, perhaps, the lungs), is such as, with existing experience, we should be warranted in adopting. Small-pox, as a type of toxic zymotic diseases, we may take first. Here are presented various phenomena which have much show of being eliminating actions, and have been pretty freely interpreted as such. The skin eruption, the salivation, the diarrhcea in children, the swelling of the face, hands, and feet, have been regarded by Sydenham and others to be processes of this kind. Of two of those mentioned, viz., the diarrhœa and ptyalism, Sydenham says, "these two evacuations being the methods that nature adopts respectively in eliminating the morbific matter." The swelling of the hands and face he calls "the pre-eminent evacuation which nature uses in this disease." The eruption is pretty generally looked upon as "necessary for the expulsion of the morbific matter." Now, there is no doubt that in small-pox the blood and tissues are leavened throughout with some toxic matter, which disorders gravely the nutrition of the various parts for which it has most affinity. Active hyperæmia and inflammation ensue in different localities, and the cell-growth in many places is variously perverted from its normal mode of life. The pulmonary and cutaneous exhalations, in fact, all that is given off from the diseased body, are toxic. It could scarcely be otherwise. After a time, this throwing off of poisonous matter ceases, and the patient is no longer a souree of infection. Clearly, he has got rid of his poison, but the question is, how? According to the language above quoted, it has been thrown off in the pustules of the skin, the sputa, the diarrhoa, and the subcutaneous effusions in face, hands, and feet. Little is said about the pulmonary exhalation, though of course it is not questioned. The more striking phenomena seem, however, to have arrested attention most. Now, it does appear to me-it long has-that there is much reason to doubt how far anything like a true elimination is accomplished by salivation, diarrhœa, cutaneous suppuration, or subcutaneous effusion. A distinction ought to be made between elimination and overflow. A cistern may run over still remaining full; and so the human system may discharge toxic matter with which it is surcharged at various parts, and yet not be thereby freed. Recovery, not merely relief, should proceed, pari passu, with true elimination. The first of the above processes coincides with more or less inflammatory irritation and swelling of the buccal mucous membrane, just as it does in mercurial ptyalism. The gland is either directly stimnlated by the abnormal matter in the blood in both cases (by variolous poison or mercury), or more probably, is excited to action, as Bernard has recently shown, by the impressions derived from the sensory nerves of the buccal membrane, which act in an inhibitory manner on its vaso-motor nerves. This is little more than stating the mere facts of the case. It is surely going a good deal farther to affirm that this process is essentially eliminative, -an effort of nature, set up as it would seem by a presiding organic agent, such as Prout imagined, to counteract the maleficent agency of disease. There is, in all probability toxic matter thrown out of the system in variolous salivation, just as some mercury may be carried out by the same channel when the drug produces severe irritation of the mouth. But it does not appear at all clearly that the flux is a benefit in either case. There is no doubt that it is very distressing to the small-pox sufferer, "ce qui l'incommode au plus haut degré et l'empêche de dormir," says Trousseau. Anyone who has suffered with a like state of mouth, though in a very minor degree, must be persuaded that the continuance of severe irritation, in so sensitive a part, for eight or nine days, must severely tax the enfeebled powers of the system. Sydenham, who looked on ptyalism as necessary, found that it was beneficial to give narcotics, though "from their power of thickening the fluids" they appeared to be contraindicated. We can well understand how much good an occasional opiate might do in the case of a patient exhausted by the torture of his sore and slavering mouth. We have no knowledge as to what amount of poisonous matter may be eliminated by the salivary channel in variola, but the analogy of mercurial salivation would not incline us to rate it highly. In the latter, mercury does not seem by any means to be invariably present; and when it is, the urine, according to Pereira, probably evacuates more, although its quantity is not of course increased at all proportionately to that of the saliva. Again, it may be remarked, that salivation does not occur in the discrete form of small-pox, a circumstance which seems not readily explicable if it is a salutary evacuation; the more so as Sydenham expressly stamps diarrhœa, which might replace it as it does in infants, as "dangerous in the distinct small-pox" (vide vol. i, p. 45, N.S.S.). There really seems to be much resemblance between the cases of variolous and mercurial salivation; and I cannot but entertain very great doubt whether either have the least claim to be regarded as beneficial efforts of nature. The only fact I find, which seems at all to favour this view, is that the abrupt cessation of ptyalism in variola is an ominous sign. Now, such cessation, especially when associated with sudden and premature subsidence of the swelling of the face, is, no doubt, the result of giving way of the vital powers, of commencing asthenia, but not its cause. Sydenham expressly states that the swelling of the face, instead of subsiding prematurely and so endangering life, is frequently, by the action of narcotics, sustained properly, and kept up until the due time that nature requires (vide p. 144, vol. i). This must surely be regarded as an instance of the toxic and supporting action of opium. Dr. Gregory, in the same way, recommends warm brandy and water, with thirty drops of laudanum, to be repeated at intervals, "when the eruptive nisus is accompanied 
with depression, and nature appears obviously unequal to the effort." It may be remarked, that salivation ceases about the fifteenth day of the disease, if not before, certainly long before all toric matter is eliminated. The same is true of the next evacuation we have to notice.

Diarrhcea, according to Trousseau, if it persist beyond the fifth day of the disease, and become violent towards the eighth, ninth, or tenth, is of bad omen. Almost all those who are thus affected die. If, however, it occurs in a patient in whom the swelling of the hands and feet does not take place towards the tenth day, it is as beneficial as in the former case it was injurious. This looks certainly very like elimination. If, however, we consider that in the swelling of the hands and feet most of the fluid effused must be reabsorbed, it still seems to me doubtful whether, even in this instance, the intestinal flux does good by elimination. Sydenham speaks very strongly of the bad effects of arresting diarrhoe in infants, many thousands, he affirms, have died in consequence. He also asserts that there is a difference in this respect between the discrete and confluent small-pox. In the former, "the evacuation is through pustules;" in the latter, "the bowels are the natural outlet by which nature seeks relief." Trousseau, who professes to take Sydenham for his guide, thinks differently. He says, p. 25, "in confluent variola diarrhœa, when it persists about the eighth, ninth, or tenth day, is a terrible phenomenon, and is to be combated by small doses of opium." Mr. Marson also directs that any undue looseness of the bowels should be arrested by chalk and opium, sulphuric acid, or other astringents. I do not think the foregoing evidence is decisive either way. It certainly does not seem to me sufficient to establish the eliminative and salutary nature of the flux.

Swelling of the face, hands, and feet, seems to be a necessary occurrence in confluent variola; the absence of the swelling in the hands and feet is declared by Trousseau to be almost always mortal. The tumefaction is connected with the maturation of the pustules; but it seems impossible that its subsidence should depend, in more than a slight degree, on the discharge from the latter. Most of the effused fluid must surely be taken up again into the blood. This phenomenon, then, which seems to be one of much more importance and necessity than the diarrhœa, can have very little eliminative effect.

The variolous eruption Dr. G. Johnson considers as " unquestionably curative," though he admits it may prove fatal by its very abundance "through its destructive influence on the skin." The chief ground for this view is, I suppose, the marked relief which is afforded to the initiatory symptoms by the appearance of the eruption, at least in the discrete form. In the confluent, Trousseau says the fever "ne se modère en rien," and there is no doubt that the amelioration which takes place when the skin becomes engaged in very much less than in the milder form. This seems opposed to the notion of the eruption being curative; for if it were, surely a more abundant outbreak should be attended, at any rate at first, with greater and not with less relief. Another point of difference is mentioned by the French observer, viz., that in discrete variola a copious perspiration sets in along with the primary fever, and continues until the period of maturation. This is usually absent when the disease is confluent. Trousseau says that it seems to constitute a favourable crisis via the skin, coming in aid, as a sort of emunction, to the great cutaneous manifestation of eruption. This may be true, although one is inclined to think that at so early a period of the disease the morbid matter can scarcely be in a fit state for expulsion. Sydenham recognises a period of separation as prior to a perioc of expulsion. I think, however, that a real elimina@ tion is much more likely to be effected through the channel of a normal secretion, than through on which is extemporised, so to speak, by a pathologicads process. It seems reasonable to believe that natura 5 organs should be more efficacious instruments than unnatural. The main reason for doubting the cor음 rectness of the view that the eruption is curative, is afforded, however, by the fact which Dr. Watson, in accordance with others, thus notices. "The object (of treatment) is to prevent, if possible, a copious eruption upon which, as we have seen, the severityand peril of the disorder entirely depend." Syden. ham had arrived at the same conclusion, as the fol $\vec{二}$ lowing extracts show. "The whole matter turnsw upon the amount of the exanthemata, and the patien lives or dies just as it is much or little." Again, "No one will wonder at the ratio between the extent" of the eruption and the danger of the disease, who considers the case of a common boil on the arm oros shoulder. The more the pus, the more the fever, since the absorption of the pus into the blood through the veins supplies the fuel of the disease Just in proportion as the physician finds the face of the patient, during the first days of the disease thick set with pustules like pinheads, he may aafelytw prognosticate that one or other of the aforesaid days death will come down upon him; the reasonf being. the violence of the secondary fever. Thisd must needs be proportionate to the quantity of pus thrown upon the blood by the innumerable aposte mes," p. 58. If the views here set forth are correct, as I believe is generally admitted, it seems to mo quite impossible to regard the cutaneous eruption aso in anywise curative. No doubt it is better that the poison should be determined to the skin, and shoulक work its mischief there, than that it should vex the brain, the cord, and the sympathetic centres, oro palsy the heart. But after all, it is only a transfer of evil from one place to another, from a more vitaf to a less vital part, and not a salutary effort of natures to remove the causa mali.

The conclusions which the above review of the sub ject seems to me to lead to, are that the morbid pro? cesses in variola are on the whole but partially and doubtfully eliminative; that they are not to be re-s garded as efforts of nature for a purpose or end, but simply as various phenomena of irritation set up byo the excess of toxic matter in one place or other, and 3 . that they afford us no indications of rational treati ment; i.e., we do not find it desirable to imitateo these processes by artiticial means.

In contrast with the above-mentioned, which, aso being essentially morbid actions, are, I think, less capable of acting therapeutically, I would place thecr pulmonic exhalation. The lungs in the healthy state present a vast surface covered with the closest pos-o sible network of capillary blood-vessels, whose delicate membrane alone separates their fluid contents 0 with the volatile matters it holds in solution from theN atmosphere. All the circulating blood courses con- $\omega$ tinually as it goes its round over this aërating sur face; and, according to a physical law, must cone stantly exchange its volatile matters for the atmospheric gases. What better apparatus could be contrived for purifying the blood from a volatile miasm ; and what more hopeful indication of treatment can astudy of all the phenomena suggest, than to maintain the pulmonary interchange between the blood and the air in the greatest activity? The providing for this is one of the great advantages of the cooling treatment.

Another very important one seems to have beeno clearly apprehended by the genius of Sydenham응 
though it has not perhaps been kept distinctly in view by subsequent observers. This is, that the subjection of the frame to any undue amount of heat so enfeebles its powers of resistance, that the same dose of poison produces a much more deleterious effect than it otherwise would. This occurs in two ways : first, by the tone of the capillaries and small vessels becoming so enfeebled that they no longer are able to retain the blood within their channels, so that extravasations occur, giving rise to purple blotches on the skin, bloody urine, and various internal congestions. I have already argued, in my Lumleian Lectures and work on Functional Nervous Disorders, that such may be the effect of heat and of all influences that impair nervous power. Secondly, the same influences probably allow a much larger quantity of morbific matter to be generated than would be in circumstances more conducive to vigour. This is well expressed in paragraph 12 of Sydenham's Epistolary Dissertation, vol. ii, p. 61, which I must ask leave to quote: "If this view be true, a blind man may see that the treatment of the first days is all important as regards the event. Inflame the spirits by cordials and bed-clothes, and their power of assimilation, already too great, becomes greater still. Add to this, that the blood and the other humours heated by such processes yield all the easier to the violent impression of the morbific particles. Hence arise more pustules than were wanted, and danger to the patient's life. Contrariwise, the opposite regimen and the free fresh air, soften down the violence of the fevered and acrid particles, and confirm and condense the humours, by strengthening them against the morbific spirits, so as to withstand their attack; whence no more variolous matter is secreted than is proportionate to the genius of the disease."

It is really remarkable to notice how Sydenham's good sense and observation guided him, in the treatment of variola, to practise contrary to what theory indicated. I must quote another passage for the sake of the bearing it has on our original subject; which my readers, I fear, will think I have forgotten. "Those who disapprove of this method, will object that by a narcotic so largely and so frequently given the peccant matter will become fixed and salivation be checked. To this I reply, that it is true that the salivation may be diminished; still it will never wholly cease. Nay more, some time after the anodyne has been given, it will break out afresh. From this accrues a double advantage. The patient, having been restored by the anodyne, is all the stronger for the expectoration. The excreted saliva, although scantier, is all the better excocted. In the next place, the decrease of the ptyalism is well made up for by the increased swelling of the hands and face. This is all the surer and all the freer for the repetition of the narcotics, especially on those days when the swelling most regularly takes place...... In respect to these, I confidently assert that no competent judge can deny that the absence of these swellings, on the days on which they are due, is a worse omen than the interruption of the ptyalism. For my own practice, I would rather risk a check to the ptyalism than a check to the swelling; and I think that this is so thoroughly required by the disease, that the practitioner who debars his patient of such an auxiliary has but little observed the complaint." (Epistolary Dissertation, vol. ii, p. 74, par. 40.)

From this passage, it is clear that Sydenham was not afraid of arresting elimination by a pretty free use of opium; and that he set more value on the due swelling of the hands and feet than on the salivary excretion, though the latter is surely more eliminant than the former. Paregorics he gave, as he tells us, once or oftener in the day, from the sirth to the seventeenth day of the disease. Another means which Sydenham used to check " the inordinate assimilation of variolous matter", was the free addition of some spirits of vitriol (sulphuric acid) to some thin drink. This "goes far to help the patient out of danger." Sulphuric acid certainly acts as an astringent to the skin, and, as most of us believe, to the bowels. My own experience convinces me that it is not eliminant.

[To be continued.]

\section{ON PROGNOSIS IN HEART-DISEASE.}

By W. H. Broddrent, M.D., Assistant-Physician to St. Mary's and the Fever Hospitals ; Lecturer on Physiology at St. Mary's Hospital Medical School.

[Continued from page 598.]

HYPERTROPHY and dilatation, then, are looked upon as caused by the valvular lesion, and as indicating its extent. The greater the degree of these structural changes, as ascertained by increased area of deep cardiac dulness, by the situation and character of the impulse and apex-beat, and by the modification of the heart-sounds, the greater the mechanical diffculty resulting from the altered valves, and the more grave the prognosis.

I must guard myself from being understood to assert without qualification the converse of thisthat the less hypertrophy and dilatation, the smaller the valvular injury. Both propositions, indeed, would be rendered more exact, if preceded by the words, "in the absence of symptoms".

'The prognostic meaning of hypertrophy and dilatation is recognised by all writers alike; but there is considerable diversity of opinion as to the relations between these changes and the valvular disease on the one hand, and subsequent effects on the circulation on the other. The idea which naturally presents itself, that the mechanical obstacle arising from the state of the valves is the cause at once of the hypertrophy and dilatation and of the derangement of the circulation, is, it seems, too simple. According to most authors, the symptoms and the ultimate fatal termination are due, not to the valvular disease itself, but to the hypertrophy and dilatation with which it is associated. Again : these structural alterations are considered by many writers as not altogether or even mainly the result of the valvular alterations, but of various accidental conditions, such as carditis, innutrition, etc., which are present in some cases and absent in others; the changes in the walls and cavities of the heart occurring or not accordingly.

It can never be altogether useless to ascertain the real relation between pathological sequences, or the connexion between pathological conditions and symptoms; and I propose to examine the two points referred to, taking first that which requires something in addition to the mechanical difficulty occasioned by the valvular lesion, as needed to give rise to changes in the muscular walls of the heart. It is stated thus by Dr. Walshe: "No direct ratio holds between the amount of hypertrophy and the amount of valvular obstruction, showing that there is something beyond the mechanical difficulty." Dr. Stokes, again, asking why in one case the cavities are unchanged, in another dilated, suggests as the cause carditis, imperfect arterialisation of the blood, obstruction of the coronary arteries, etc. It is not 